\title{
EVIDENCIAS DE (INTER)SUBJETIVIDAD EN EL DISCURSO INSTITUCIONAL-ESTATAL: LA CONTRIBUCIÓN DEL ANÁLISIS DEL DISCURSO PARA EL PENSAMIENTO CRÍTICO
}

\author{
(Evidence of inter(subjectivity) in institutional-government \\ discourse: the contribution of discourse analysis \\ to critical thinking)
}

\author{
Mariana Carolina Marchese ${ }^{1}$ \\ (Universidad de Buenos Aires-UBA/CONICET)
}

\begin{abstract}
Within a Critical Discourse Analysis framework, with a qualitative methodology, and Discourse Analysis as a method, this paper presents linguistic evidence how allegedly objective texts are, in fact, construed (inter) subjectively. Results show two series of resources which act jointly. One series of resources aims at eliminating the speaker from the surface of the text, while the other is formed by evaluativesubjective resources. Thus, Discourse Analysis enables critical thinking as regards the complex role discourse plays in social events.
\end{abstract}

Keywords: institutional-government discourse, objectivity, subjectivity, Critical Discourse Analysis.

\section{RESUMEN}

Desde el marco teórico del Análisis Crítico del Discurso, a partir de una metodología cualitativa y a través del Análisis del Discurso como método, este trabajo presenta evidencias lingüísticas de que aquellos textos que se suponen objetivos son construidos (inter)subjetivamente. Los resultados del análisis manifiestan dos

1 Profesora de la carrera de Letras de la Facultad de Filosofía y Letras de la UBA. Participa en el proyecto UBACyT F127, Análisis crítico del discurso de la pobreza extrema urbana en la Argentina y América Latina en la posmodernidad. Una aproximación a la Red Latinoamericana de Análisis del Discurso de la pobreza, dirigido por la Dra. María Laura Pardo. Es becaria del Consejo Nacional de Investigaciones Científicas y Técnicas (CONICET) y desarrolla su investigación de Tesis Doctoral, bajo la dirección de la Dra. Pardo y la co-dirección de la Dra. Massone. 
series de recursos que actúan en forma conjunta. Una serie de recursos que apuntan a borrar al emisor de la superficie textual y otra serie, que actúa en forma paralela a la anterior, formada por recursos evaluativos-subjetivos. Así, el Análisis del Discurso permite el pasaje a la reflexión crítica sobre el complejo rol del discurso dentro de los fenómenos sociales.

Palabras-clave: discurso institucional-estatal, objetividad, subjetividad, Análisis Crítico del Discurso.

\section{Introducción}

El discurso de las instituciones que conforman el aparato estatal tradicionalmente se ha manifestado a través de géneros que se suponen objetivos y, en este sentido, imparciales respecto de los contenidos que abordan. La formulación de la ley es un claro ejemplo de esta pretensión de objetividad que olvida que el uso del lenguaje es una actividad inherentemente humana y que es imposible que los seres humanos, en tanto tales, podamos despojarnos absolutamente de nuestra subjetivad al hacer uso de esta herramienta de pensamiento y, paralelamente, de comunicación. Por esta razón, el objetivo de este trabajo es exponer evidencias lingüísticas de marcas de subjetividad relevadas dentro del discurso institucional- estatal, para demostrar cómo la objetividad no existe en sí misma, sino que es una construcción (inter)subjetiva que contribuye a naturalizar posiciones ideológicas.

Dado que mis intereses siempre han estado vinculados a las temáticas de la pobreza extrema y de la exclusión social, he seleccionado un género específico para analizar. Se trata de informes de relevamiento de casos sobre sujetos en situación de extrema pobreza, producidos por trabajadores sociales, psicólogos y sociólogos, dentro de un programa del Gobierno de la Ciudad Autónoma de Buenos Aires. Este trabajo forma parte del propósito general de mis investigaciones que apunta a que los resultados obtenidos colaboren a mejorar nuestra comprensión e interpretación crítica del complejo rol del discurso dentro de los fenómenos sociales y, asimismo, que los resultados de estos análisis 
lingüísticos conjuntamente con sus interpretaciones en el plano social se conviertan en una herramienta para disciplinas afines y en un instrumento para apoyar la discusión, basada en datos lingüísticos concretos, sobre la elaboración de políticas públicas que involucran a sujetos en situación de extrema pobreza.

\section{La extrema pobreza en la Ciudad Autónoma de Buenos Aires}

Durante los últimos años el concepto de posmodernidad se ha convertido en un protagonista indiscutido de los análisis sobre la sociedad y la cultura. En la línea de Jameson, entiendo dicho concepto no sólo como un fenómeno estético, sino como la pauta cultural dominante de la lógica del capitalismo avanzado (Jameson, 2005: 101). Este capitalismo avanzado o neoliberalismo se ha concretizado en la aparición de políticas de diversos tipos que sacralizan el mercado multinacional por encima de las leyes de los Estados nacionales. Boron (2003) plantea, desde el marco de la sociología aplicada a la realidad latinoamericana, que la minimización del accionar del Estado dentro de un mercado globalizado produce una sociedad en la cual los derechos de los ciudadanos son redefinidos desde una lógica mercantil. De modo similar, Sklair (2003) indica que el accionar de las corporaciones trasnacionales generó graves consecuencias no sólo a nivel económico, sino también político, social y cultural.

Particularmente, respecto del caso de Argentina dentro de este contexto mundial, los trabajos de Fidel (2004) y de Mingo et al. (2006) coinciden en que el discurso neoliberal se instaló en la vida social y cultural de nuestro país durante la década del noventa. Explican que si bien durante el primer lustro de esa década la economía parecía crecer positivamente controlándose el proceso inflacionario, en 1995 la dependencia de la inversión especulativa externa comenzó a mostrar sus debilidades. Estas se manifestaron en el incremento de la tasa de desocupación, en el consecuente aumento de la pobreza estructural, en la aparición de los 
llamados nuevos pobres y en un proceso de desarticulación del aparato estatal que trajo aparejada una redefinición de la interacción entre el Estado y los sujetos sociales. Aunque durante los años 1996 a 1998 la economía siguió describiendo un proceso de crecimiento, este no logró revertir la agudización de la pobreza. Fidel (2004) y Mingo et al. (2006) coinciden en señalar que justamente en ese periodo la inequidad de la distribución de la riqueza comenzó a tornarse crítica.

Dentro de este contexto político-económico, social y cultural, quienes habitamos la Ciudad de Buenos Aires pudimos observar que durante la última década del siglo XX y principios del siglo XXI se agudizó la yuxtaposición de las imágenes de la pobreza y de la riqueza (Pardo, 2008a) y que estas realidades, en la mayoría de los casos, no coexisten pacíficamente. Como explica Bauman:

...el territorio urbano se convierte en el campo de batalla de una guerra continua por el espacio, que a veces estalla en espectáculo público... [...] Las fortificaciones construidas por la elite y la autodefensa por medio de la agresión practicada por los excluidos se refuerzan mutuamente...

(Bauman, 1999: 33)

Las consecuencias del modelo neoliberal se concretizaron, también, en la aparición de lo que el antropólogo Augé (1993) denominó no-lugares o espacios de anonimato. Siguiendo a Sarlo (1994), el shopping es una escena de estos espacios que definen y exhiben la lógica neocapitalista de la posmodernidad que, al debatirse entre la abundancia y la pobreza, también produce no-lugares marginales, como casas tomadas y casas extendidas-fragmentadas en donde cohabitan varias familias. Krmpotic y Allen (2003) coinciden con Reynals y Redon (1991) en afirmar que el incremento de estos modos de habitar la ciudad se relaciona con la emigración de pobladores del interior del país y de Latinoamérica, respecto de sus lugares de origen, debido a la desocupación y a la subocupación relacionadas con la aplicación de nuevas políticas laborales. 
Estos trabajos postulan que esta historia de marginalidad pone al desnudo una estructura social que se caracteriza por la fragmentación y polarización creciente entre algunos sectores de la población cada vez más ricos y otros cada vez más pobres. En concordancia, Malanca y Repetto (2003), respectivamente desde la psicología y el trabajo social, indican que durante el segundo lustro de la década del noventa se agudizaron progresivamente las demandas asociadas con las problemáticas sociales de las personas en situación de pobreza y agregan que el fenómeno de los "sin techo" se hizo visiblemente masivo y notable en las calles de la Ciudad de Buenos Aires en el transcurso de ese lustro, dado que estas personas quedaron desanudadas de la red social. Como expongo en trabajos anteriores (Marchese, 2008a, 2008b, en prensa a y en prensa b), la problemática social alcanzó un extremo tal que, tanto a nivel nacional como jurisdiccional, el Estado al elaborar políticas públicas puso en circulación frases nominales como emergencia pública, emergencia laboral y emergencia habitacional.

\section{Marco teórico-metodológico}

Esta investigación se posiciona dentro del paradigma interpretativista (Guba y Lincoln, 1998), se inscribe en el marco teórico del Análisis Crítico del Discurso (ACD) (Fairclough, 1992 y 1995; van Dijk ,1993; Resende y Ramalho, 2006; Pardo Abril, 2007 y Pardo, 2008b) y la metodología es cualitativa (Wodak y Meyer, 2003 y Pardo, 2008b). Asimismo, debido a que se trata de un estudio de caso, las conclusiones obtenidas no son generalizables, ni universales.

En la línea de Fairclough (1992), entiendo que todo hecho comunicativo, analíticamente puede estudiarse a través de la teorización de tres prácticas (textual, discursiva y social) que en la comunicación concreta y cotidiana se relacionan dialécticamente y, de hecho, son indisolubles, porque los textos (práctica textual) se producen dentro de una matriz genérica (práctica discursiva) y los géneros son construcciones inherentemente sociales y humanas que surgen en la comunicación (práctica social). 
A partir de lo expuesto en el párrafo anterior y de que la metodología es cualitativa, razón por la cual se busca una aproximación lo más inductiva posible respecto de los textos, el corpus será analizado a través de teorías de índole cualitativa. A su vez, dichas teorías permiten observar la relación dialéctica entre las prácticas textual, discursiva y social. Para el análisis de la práctica textual utilizo la Teoría de la Valoración (White, 1999 y Kaplan, 2004) y la Teoría de la Tonalización (Lavandera, 1985 y Pardo, 1996). Para el estudio de la práctica discursiva me baso en el modelo de SPEAKING (Hymes, 1986) como teoría de medio rango, especialmente en el componente Género. Finalmente, para interpretar cómo operan los datos del análisis anterior en el plano social en términos ideológicos, he realizado lecturas de diferentes disciplinas.

El corpus está compuesto por quince informes de relevamiento de casos sobre sujetos en situación de pobreza extrema, realizados durante el periodo 2001 a 2003. Estos informes fueron producidos por profesionales del campo de las ciencias humanas que trabajaban dentro de un programa del Gobierno de la Ciudad Autónoma de Buenos Aires cuyo núcleo de acción era la denominada emergencia social y, sobre todo, la emergencia habitacional que se encontraba comprendida dentro de la primera. Los profesionales (trabajadores sociales, psicólogos y sociólogos) realizaban los informes luego de los denominados relevamientos en la vía pública, durante los cuales entrevistaban a personas y familias en situación de calle. El objetivo de estos informes era dejar asentada la información más relevante sobre el caso y plantear estrategias posibles para resolver la situación de los sujetos. Además, dado que estas podían relacionarse, por ejemplo, con la derivación a otros programas sociales, con la búsqueda de algún familiar o con la solicitud de intervención de la justicia civil, los informes circulaban no sólo dentro del programa, sino también en otros ámbitos. Otro dato importante en función del análisis es que los informes eran producidos por el profesional o los profesionales que habían llevado a cabo el relevamiento.

Para circunscribir el corpus, se utilizaron sólo informes correspondientes a casos de sujetos adultos y se eliminaron aquellos 
relacionados con familias en situación de calle. Esto se debió a que en este último tipo de informes se cruza la problemática de la pobreza extrema con la de los menores en situación de calle lo que conduciría a otro tipo análisis que probablemente abordaré en próximos trabajos.

Antes de comenzar el análisis, quisiera realizar algunas aclaraciones respecto de la elección de las teorías de la Tonalización y de la Valoración para el análisis de la práctica textual. En la línea de Pardo considero que resulta muy útil trabajar con estas teorías en forma conjunta para abordar un análisis como el que propongo en este artículo.

De la Teoría de la Valoración utilicé, principalmente, aquellos elementos que se encuentran dentro del sistema de actitud y que permiten estudiar la expresión lingüística del juicio. Así, mediante el subsistema de actitud-juicio, analizo cómo a través de ciertos recursos lingüísticos se evalúa el comportamiento de los sujetos en situación de pobreza extrema a través de juicios relacionados con una estimación social y con una sanción social negativas ${ }^{2}$. Esta teoría presenta, también, el subsistema de gradación que atraviesa el subsistema de actitud-juicio y que, de hecho, puede considerarse como una tonalidad a través de todo el sistema de la valoración (White, 1999) Ahora bien, puesto que entiendo que los modos de tonalizar de cada lengua son particulares y dado que disponemos de una descripción de dichos recursos para el español, he considerado apropiado realizar este análisis a partir de los recursos de mitigación y de refuerzo propios del español que se encuentran dentro de lo que Pardo (1996) denomina Tonalización. Estos recursos amplían los propuestos por Lavandera (1985) como recursos de mitigación presentes en el uso del español. Además, tal y como podemos observar en Pardo (1996), los recursos lingüísticos utilizados para reforzar (aumentar la carga semántica de un ítem léxico) y para mitigar (restarle carga semántica a un ítem léxico) son nociones que siempre se relacionan con el género en el que se insertan.

2 Cabe mencionar que la Teoría de la Valoración no está pensada desde el español. Sin embargo, el proceso de análisis que se efectuó para este trabajo no presentó inconvenientes. 
Por otro lado, estas dos teorías en tanto herramientas metodológicas se apoyan en fundamentos teóricos similares. La Teoría de la Valoración, derivada de la lingüística sistémico-funcional, busca profundizar el estudio de la función interpersonal con el fin de describir y explicar los sistemas de opciones semánticas que ofrece el lenguaje para evaluar, adoptar posiciones... construir personas textuales, y lograr que las posturas ideológicas parezcan "naturales" (Kaplan, 2004: 52), mientras que la Teoría de la Tonalización pretende explicar cómo los recursos lingüísticos utilizados en un texto para mitigar y para reforzar dan cuenta de la postura o del punto de vista del emisor frente a los contenidos que aborda. Asimismo, ambas teorías consideran que la dimensión dialógica es fundamental para la construcción del significado de las representaciones socio-discursivas que poseen los sujetos sociales.

Por último, también en concordancia con Pardo, quisiera agregar que aunque ambas teorías presentan las similitudes mencionadas, considero que la Teoría de la Valoración se inclina hacia el estudio léxico-gramatical de los textos, mientras que la Teoría de la Tonalización se orienta no sólo hacia dicho estudio, sino que también provee herramientas para analizar cómo mediante la sintaxis se construyen significados sociales.

\section{La construcción lingüística de la objetividad en el discur- so institucional-estatal}

Siguiendo a Bajtín (1982), considero el concepto de géneros discursivos como tipos temáticos, composicionales y estilísticos de enunciados determinados y relativamente estables que se vinculan a esferas de uso del lenguaje. Asimismo, en palabras de Bajtín, los géneros discursivos son correas de transmisión entre la historia de la sociedad y la historia de la lengua (Bajtín, 1982: 254). De modo similar, Hymes (1986) indica que la noción de género implica la posibilidad de identificar características formales definidas y reconocidas por la tradición. 
Las instituciones públicas que constituyen el aparato estatal históricamente se han manifestado a través de géneros discursivos que despliegan una serie de características formales relativamente estables que están definidas y son reconocidas por la tradición. Es decir, en estas instituciones, los textos deben ser producidos siguiendo pautas específicas. Estas pautas están disponibles, en tanto sistema de opciones potenciales, dentro de las posibilidades que la lengua ofrece y se convierten en recursos cuando se hace uso de ellas (Menéndez, 2005). Es en ese proceso que se seleccionan algunas opciones y se descartan otras. De esta manera, el discurso institucional-estatal se convierte en un ámbito de la acción social que implica el uso convencional y pautado de ciertas formas lingüísticas y la exclusión de otras. Para este fin, existen, por ejemplo, modelos de textos a seguir y también se suelen dictar cursos en las respectivas áreas de capacitación de personal de las diferentes instituciones. Dar cuenta de esos recursos lingüísticos que presentan alta frecuencia de uso en los textos y que, en este sentido, se convierten en elementos no-marcados o de uso común dentro del género (Pardo, 1996) resulta importe en ese trabajo, porque muchos de esos elementos lingüísticos apuntan a borrar o a mitigar la presencia del emisor de la superficie textual, o sea, la finalidad del uso de estos recursos es lograr la producción de textos con características de objetividad e imparcialidad y despojados de subjetividad.

Durante algunos años, me desempeñé como docente de capacitación en diferentes instituciones del Gobierno de la Ciudad Autónoma de Buenos Aires y resulta interesante destacar que aunque en los últimos años ha surgido una suerte de proceso de revisión respecto de los elementos que conforman el discurso institucional-estatal, la pauta referida al borramiento del emisor sigue manteniéndose como uno de sus pilares. Asimismo, aunque los textos producidos dentro del género discursivo institucional-estatal y del género discursivo legal-normativo (leyes, decretos, etc.) difieren en su función o ámbito de acción social (Wodak, 2003), ambos comparten ciertos recursos lingüísticos orientados hacia la pretensión de objetividad. Si bien el estudio de las diferencias y de las similitudes entre dichos géneros, 
como parte de un continuum al que podríamos denominar discurso del Estado, forma parte de una investigación en desarrollo, entiendo que resulta pertinente realizar esta aclaración, debido a que, como indiqué, el uso de ciertos recursos lingüísticos ligados a la búsqueda de objetividad parecería ser, dada su alta frecuencia de uso, uno de los elementos más importantes dentro de este continuum discursivo mediante el cual el Estado se comunica con los ciudadanos y, a su vez, a través del cual las instituciones estatales se comunican entre sí. A continuación, presento los recursos lingüísticos predominantes utilizados para mitigar o borrar la presencia del emisor y los comparo con otras opciones posibles dentro del sistema de la lengua que son desechadas a favor de los primeros.

Tabla 1. Recursos lingüísticos para la construcción de objetividad

\begin{tabular}{|l|lll|}
\hline Recursos lingüísticos utilizados & Otras opciones posibles no utilizadas \\
\hline Verboides (infinitivo, participio y gerundio) & Verbos conjugados & \\
\hline Nominalizaciones & Verbos conjugados & \\
\hline $\begin{array}{l}\text { Voz pasiva (pasiva con se, pasiva perifrástica } \\
\text { con complemento agente omitido y } \\
\text { participio pasado con auxiliar ser omitido) }\end{array}$ & Voz activa & \\
\hline $\begin{array}{l}\text { Pronombre se con valor impersonal y verbos } \\
\text { impersonales }\end{array}$ & Voz activa & \\
\hline $\begin{array}{l}\text { Emisor en primera persona plural o en } \\
\text { tercera singular }\end{array}$ & $\begin{array}{l}\text { Emisor en primera persona del } \\
\text { singular }\end{array}$ & \\
\hline $\begin{array}{l}\text { Emisor construido como agente colectivo } \\
\text { Emisor en primera persona del }\end{array}$ & $\begin{array}{l}\text { dingular } \\
\text { smisor construido como complemento }\end{array}$ & $\begin{array}{l}\text { Emisor en primera } \\
\text { singular }\end{array}$ & \\
\hline $\begin{array}{l}\text { Emircunstancial de lugar } \\
\text { circona del }\end{array}$ & & \\
\hline
\end{tabular}

Los informes de relevamiento de casos que componen el corpus presentan alta frecuencia de aparición de los recursos lingüísticos expuestos en la Tabla 1. Por ejemplo: 


\section{Uso de verboides}

Ej. 1: Respondiendo al llamado de la vecina Cristina, quien pide asistencia para un señor que se encuentra en la entrada de su edificio.

Ej. 2: Siendo las 02.30 hs. AM, se concurre al lugar citado, en Santa Fe 2540, contactándose a un hombre...

Ej. 3: Considerando el estado del causante su desequilibrio mental y la presuntiva cronicidad del caso, se estima conveniente efectuar la derivación...

\section{Uso del participio pasado con auxiliar ser y agente omitidos}

Ej. 4: De la entrevista mantenida surgen indicadores...

Ej. 5: Dado el grado de cronicidad que presentan las personas evaluadas y debido a su cuadro de alcoholismo se considera pertinente... la realización de un tratamiento...

Uso de pasiva perifrástica con complemento agente omitido

Ej. 6: ... la persona de referencia fue entrevistada.

\section{Uso de pasiva con $s e$}

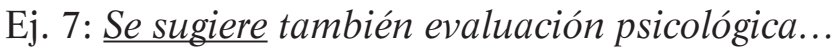

Ej. 8: Allí se realiza contacto con un señor de avanzada edad...

Ej. 9: Por solicitud de la Sra. A. (Cámara de Diputados de la Nación) se visita el sitio en cuestión...

Ej. 10: ... se decide pedir asistencia en la comisaría 18...

\section{Uso del pronombre se con valor impersonal}

Ej. 11: Al respecto se lo interroga sobre el paradero (de la institución) y no sabe precisarlo. 
Ej. 12: Por lo tanto, se evalúa al Sr. HD...

Ej. 13: Por lo consiguiente se lo evalúa...

Emisor construido en tercera persona singular

Ej. 14: ... el móvil parte hacia el primer punto de referencia.

Ej. 15: ...el móvil se dirige al lugar de referencia...

\section{Huellas de subjetividad en el discurso institucional-estatal}

Paralelamente a los recursos descriptos, utilizados para mitigar la presencia de los emisores de los textos, el análisis del corpus reveló la presencia de otra serie de recursos que son predominantemente evaluativos en tanto que son juicios de valor y, como tales, evidencian la subjetividad de los emisores de estos textos. Estos recursos, en principio, serían marcados dentro del género discursivo institucional-estatal, es decir, no comunes o no esperables dentro de él. Sin embargo, el análisis evidenció que presentan alta frecuencia de aparición (surgen en el 100 $\%$ de los informes que componen el corpus). A partir de ello, planteo el interrogante de si estos recursos evaluativos no serían la característica específica de estos informes. Así, estos recursos evaluativos diferenciarían a los informes de casos aquí analizados como un subgénero discursivo específico dentro del género discursivo institucional-estatal. Sin embargo, si esto fuera así, entraríamos en una contradicción, puesto que dentro un género que se supone objetivo tendríamos, como característica específica de uno de sus subgéneros, marcas de subjetividad y de evaluación, por ende de parcialidad. A no ser que aceptemos que la objetividad es sólo una ilusión o un efecto originado por recursos lingüísticos que intentan esconder la subjetividad de los emisores de los textos.

White (1999) propone una clasificación dentro del subsistema de actitud-juicio, que es aquel mediante el cual se evalúa el comportamiento humano, formada por evaluaciones que giran en torno a las categorías de estimación social y de sanción social. Estas pueden ser positivas o 
negativas y, a su vez, se dividen en subcategorías. La categoría estimación social posee como subcategorías: normalidad, capacidad y tenacidad y la categoría sanción social presenta como subcategorías: veracidad y adecuación. Asimismo, las evaluaciones pueden ocurrir en los textos en forma explícita (inscripta) o implícita (evocada).

Tabla 2. Subsistema de actitud-juicio

\begin{tabular}{|l|l|l|}
\hline ESTIMACIÓN SOCIAL & $\begin{array}{l}\text { POSITIVA } \\
\text { (ADMIRAR) }\end{array}$ & $\begin{array}{l}\text { NEGATIVA } \\
\text { (CRITICAR) }\end{array}$ \\
\hline $\begin{array}{l}\text { Normalidad: ¿es el } \\
\text { comportamiento de la persona } \\
\text { inusual o habitual y corriente? }\end{array}$ & $\begin{array}{l}\text { normal, común, } \\
\text { afortunado, etc. }\end{array}$ & $\begin{array}{l}\text { raro, desafortunado, } \\
\text { excéntrico, vetusto, etc. }\end{array}$ \\
\hline $\begin{array}{l}\text { Capacidad: ¿es la persona } \\
\text { competente, capaz? }\end{array}$ & $\begin{array}{l}\text { apto, inteligente, fuerte, } \\
\text { sano, cuerdo, etc. }\end{array}$ & $\begin{array}{l}\text { tonto, lento, torpe, } \\
\text { débil, demente, etc. }\end{array}$ \\
\hline $\begin{array}{l}\text { Tenacidad: ¿es la persona } \\
\text { confiable, bien dispuesta, } \\
\text { tiene decisión? }\end{array}$ & $\begin{array}{l}\text { cumplidor, responsable, } \\
\text { infatigable, resuelto, } \\
\text { perseverante, etc. }\end{array}$ & $\begin{array}{l}\text { poco confiable, } \\
\text { irresponsable, perezoso, } \\
\text { poco perseverante, etc. }\end{array}$ \\
\hline $\begin{array}{l}\text { SANCIÓN SOCIAL } \\
\text { POSITIVA } \\
\text { (ALABAR) }\end{array}$ & $\begin{array}{l}\text { NEGATIVA } \\
\text { (CONDENAR) }\end{array}$ \\
\hline $\begin{array}{l}\text { Veracidad: ¿es honesta la } \\
\text { persona? }\end{array}$ & $\begin{array}{l}\text { honesto, sincero, veraz, } \\
\text { verosímil, creíble, } \\
\text { auténtico, etc. }\end{array}$ & $\begin{array}{l}\text { embustero, mentiroso, } \\
\text { deshonesto, etc. }\end{array}$ \\
\hline $\begin{array}{l}\text { Adecuación: ¿la conducta } \\
\text { de la persona es ética o } \\
\text { reprochable? }\end{array}$ & $\begin{array}{l}\text { bueno, moral, } \\
\text { obediente a la leyes, } \\
\text { etc. }\end{array}$ & $\begin{array}{l}\text { malo, inmoral, brutal, } \\
\text { etc. }\end{array}$ \\
\hline
\end{tabular}

El corpus que analizamos aquí evidencia la presencia de recursos lingüísticos a través de los cuales los sujetos en situación de extrema pobreza son evaluados en forma negativa. Específicamente, los juicios comprendidos dentro de la categoría estimación social negativa están vinculados a evaluaciones según las cuales la persona en situación de pobreza extrema es asociada con una estimación baja o negativa dentro de su comunidad y, en este sentido, se los presenta como disfuncionales dentro de ella. Los juicios que se encuentran dentro de la categoría de sanción social negativa tienen 
que ver con regulaciones propias de una cultura o de una comunidad. Estas regulaciones pueden estar codificadas explícitamente a través de leyes o ser de tipo más implícito, por ejemplo, regulaciones morales que circulan en una comunidad y que subyacen en esta como creencias tácitas. Dado que a los sujetos en situación de pobreza extrema se los presenta discursivamente como contraventores de estas normas legales y morales que regulan la vida en sociedad, también reciben una condena legal o moral.

A continuación, presento los ejemplos más representativos de aquellos recursos lingüísticos a través de los cuales se construye la evaluación negativa de los sujetos en situación de pobreza extrema. Para la ejemplificación, sigo la división según las categorías y subcategorías mencionadas e indico si corresponden a juicios inscriptos o evocados.

\section{Estimación social negativa inscripta}

\section{Falta de normalidad}

Adjetivos que expresan deterioro físico (resaltados con subrayado) que suelen co-ocurrir con adverbios que refuerzan el concepto de deterioro (resaltados en cursiva):

Ej. 16: Se presenta desaliñado...

Ej. 17: El señor exhibe rastros que denotan cronicidad en situación de calle, se lo nota ciertamente deteriorado.

Ej. 18: Aspecto físico sumamente deteriorado...

Sustantivos que expresan deterioro físico (resaltado con subrayado) en co-ocurrencia con adjetivos que refuerzan el concepto de deterioro (resaltado en cursiva):

Ej. 19: Respecto a su presencia, se denota... menoscabo físico considerable...

\section{Falta de capacidad}

Sustantivos y adjetivos derivados de formas positivas a través de prefijos que significan privación o falta (resaltado en cursiva), utilizados 
para expresar incapacidad física o escasa capacidad de desenvolvimiento e inserción social:

Ej. 20: El señor sería analfabeto...

Sustantivos que expresan incapacidad física (resaltado con subrayado) en co-ocurrencia con adjetivos que refuerzan dicha incapacidad (resaltado en cursiva):

Ej. 21: ... se lo evalúa en extrema vulnerabilidad, agravada por su imposibilidad para trasladarse...

Estimación social negativa evocada

Aquí los juicios de valor son activados a través de términos aparentemente neutrales y los recursos que materializan estos juicios evocados desplazan la figura de los sujetos en situación de pobreza extrema desde el concepto de "vulnerabilidad" hacia el de "problemas mentales". Se trata de juicios evocados, porque aunque no explicitan que la persona en situación de pobreza extrema sea, por ejemplo, irresponsable o perezosa, lo dejan inferir a través de recursos lingüísticos. Estos juicios aparecen en el nudo y en el cierre de los informes, mientras que los inscriptos se encuentran en la zona introductoria de los textos. Tal vez, algunos de estos juicios evocados podrían ser caracterizados como inscriptos dentro del tecnolecto de la psiquiatría. Sin embargo, como indiqué, este tipo de informes circulan en diferentes ámbitos y son leídos por receptores con diversos bagajes. Por ello, consideré más adecuado clasificarlos como evocados.

\section{Falta de capacidad}

Adjetivos y sustantivos (resaltados con negrita) derivados de formas positivas a través de prefijos que significan negación (resaltados en cursiva). A su vez, los adjetivos suelen co-ocurrir con adverbios cuyo significado o carga semántica refuerza el concepto de incapacidad mental (resaltados con subrayado simple):

Ej. 22: Se encuentra desorientado témporo-espacialmente... 
Ej. 23: ...se encuentra perdido y en condiciones de indefensión...

Ej. 24: Se encuentra desorientado temporalmente...

Adjetivos (resaltados con subrayado) derivados de formas positivas a través de prefijos que significan privación o falta (resaltados en cursiva), asignados al discurso del sujeto.

Ej. 25: Su discurso es por momentos incoherente e incompresible sobre todo en lo que se refiere a fechas y lapsos de tiempo...

Ej. 26: ... su discurso es incoherente...

Adverbios mediante los cuales se mitiga la competencia mental del sujeto (resaltados en negrita), en el caso de que esta sea expresada positivamente (se subrayan las formas positivas):

Ej. 27: Su discurso se desarrolla de manera coherente aunque su dirección resulta poco clara...

Ej. 28: Según se observa impresiona estar parcialmente orientado auto y alopsiquicamente...

\section{Falta de tenacidad}

Verbos referidos a la acción de trabajar que co-ocurren con el adverbio negativo no (resaltados con subrayado). Estos verbos negados, a su vez, co-ocurren con adverbios o construcciones adverbiales de tiempo que refuerzan la falta de tenacidad del sujeto (resaltadas en cursiva):

Ej. 29: ...no trabaja desde hace más de tres años.

Ej. 30: ... expresa no haber trabajado nunca, estar sin hacer nada...

\section{Sanción social negativa inscripta}

\section{Falta de adecuación}

Este tipo de sanción se expresa a través de adjetivos relacionados con la problemática del alcoholismo y con la violencia: 
Ej. 31: El señor de referencia... sería alcohólico...

Ej. 32: El mismo se encuentra ebrio...

Ej. 33: Allí se contacta a la Sra. B. (tel:XXXX) quien afirma ser hija del Sr. B. y a quien señala como padre abandónico y golpeador.

\section{Sanción social negativa evocada}

\section{Falta de la veracidad}

Uso del condicional simple a través del cual se pone en duda el discurso del sujeto en situación de pobreza (resaltados con subrayado). Este uso contrasta en el co-texto con el uso del modo indicativo (resaltados en cursiva) para referirse al discurso de otros actores que aparecen en los textos, como vecinos de la ciudad o familiares de la persona en situación de pobreza extrema. Este es el caso del ejemplo (33) citado nuevamente a continuación:

Ej. 33: Allí se contacta a la Sra. B. (tel:XXXX) quien afirma ser hija del Sr. B. y a quien señala como padre abandónico y golpeador.

Ej. 34: Según expresan los vecinos, el mismo se instala en el lugar por las noches...

Ej. 35: Manifiesta que se encontraría en calle junto a su marido, ausente al momento de la entrevista. El mismo se llamaría HD... y sería excombatiente de Malvinas... Ambos se encontrarían desocupados actualmente.

Ej. 36: Ella y el antes mencionado venderían la revista...

\section{La objetividad como producto de la inter(subjetividad)}

Como mencioné, los informes de caso comparten con otros textos propios del discurso del Estado una serie de recursos lingüísticos ligados 
a la construcción de la objetividad. Sin embargo, como se puede apreciar en la Tabla 3, esos recursos coexisten, en ocasiones de un modo casi paradójico, con recursos evaluativos que se convierten en huellas que evidencian que existe algún tipo de subjetividad detrás de estos textos. La pregunta que me formulo en este punto del análisis es: ¿cómo se construye esa subjetividad?

\section{Tabla 3. Coexistencia de recursos}

\begin{tabular}{|l|}
\hline $\begin{array}{l}\text { Construcción discursiva de la } \\
\text { objetividad }\end{array}$ \\
\hline Uso de verboides \\
\hline $\begin{array}{l}\text { Uso del participio pasado con auxiliar } \\
\text { "ser” y agente omitidos }\end{array}$ \\
\hline $\begin{array}{l}\text { Uso de pasiva perifrástica con } \\
\text { complemento agente omitido }\end{array}$ \\
\hline Uso de pasiva con se \\
Emisor construido en tercera persona \\
singular \\
impersonal pronombre se con valor \\
\hline
\end{tabular}

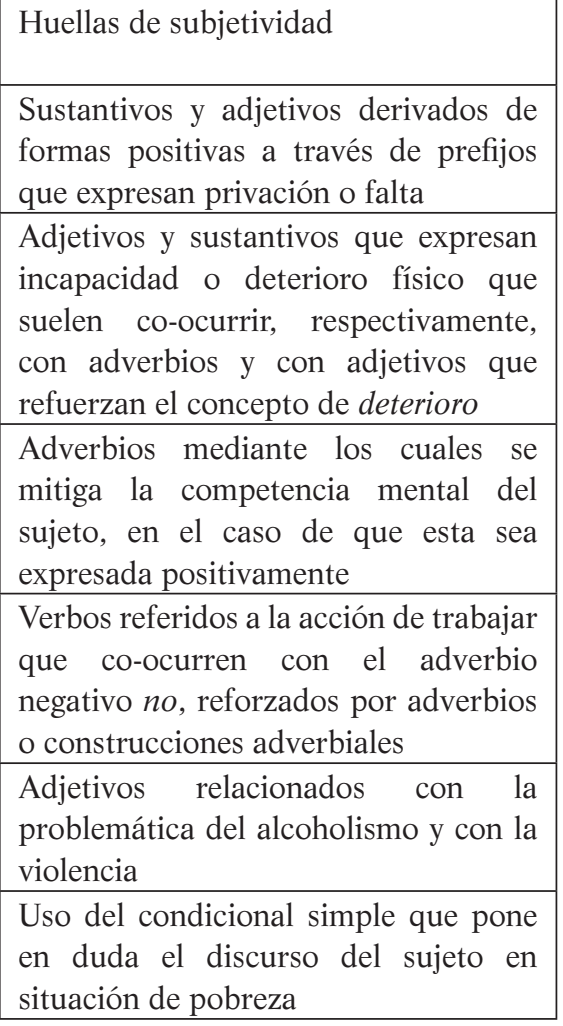

Hace algunos años investigo la relación entre el Estado y los sujetos en situación de pobreza extrema, así como la construcción de la figura de los segundos en el discurso del primero. Hasta el momento, todas 
mis investigaciones, abordando diferentes corpus y mediante diferentes métodos y teorías de análisis, han evidenciado resultados similares. Además, durante estas investigaciones he participado en actividades desarrolladas en el marco del Gobierno de la Ciudad Autónoma de Buenos Aires, en donde también trabajé varios años. A través de estas experiencias, pude apreciar que los profesionales de diversos campos de las humanidades cuyo trabajo está dedicado a sujetos que atraviesan situaciones de extrema pobreza no son explícitamente prejuiciosos respecto de ellas en sus diálogos laborales cotidianos extraoficiales. Quiero decir que los resultados del análisis de este corpus evidencian más bien que las evaluaciones son parte de un sistema por naturaleza prejuicioso que obliga a quienes forman parte de él a enmarcarse dentro de sus pretensiones y lineamientos de un modo no coercitivo. Esta acción no coercitiva resulta aún más peligrosa que una acción explícitamente represiva, porque de esa manera el prejuicio se internaliza mediante un proceso lento y sutil que se lleva a cabo en una suerte de pacto implícito e (inter)subjetivo a través de prácticas discursivas inherente humanas como la que aquí he analizado que históricamente han pretendido ser objetivas cuando no lo son. De este modo, en el plano social (es decir, en y a través de las prácticas textuales y discursivas cotidianas), implícitamente se naturalizan posiciones ideológicas que se van construyendo (inter)subjetivamente. $\mathrm{Si}$ bien en este trabajo no abordé en profundidad el análisis del subsistema de compromiso propuesto por la Teoría de la Valoración, en el corpus pueden observarse algunas emisiones que ponen en evidencia la existencia de otras voces posibles. Por ejemplo:

a) Presuntivamente se trata de un cuadro confusional.

b) Se observan manierismos y estereotipias verbales.

Si comparamos estas dos emisiones, la emisión a) podría ser clasificada dentro de la teoría como un caso de heteroglosiaintravocalizada, mientras que b) sería una emisión monoglósica. Más allá de la clasificación en sí misma y de que en la línea de Bajtín (1982) 
considero que el lenguaje es inherentemente dialógico, lo que me interesa resaltar es que la aparición de adverbios como presuntivamente abre la posibilidad de otra voz dentro un discurso en donde, históricamente, ha predominado una voz que juzga negativamente a todo aquel que por motivos diversos esté ubicado en los márgenes del sistema. De hecho, desde el subsistema de compromiso, varios de los adverbios analizados en este trabajo podrían clasificarse también como recursos mediante los cuales los emisores de los textos "dialogan” con otras voces posibles, ya sea para cerrar o para abrir "la discusión” con ellas.

\section{Consideraciones finales}

Por último, quisiera destacar que este trabajo también ha intentado dar cuenta de que el análisis del discurso puede actuar como complemento del análisis de otras ciencias sociales, porque hace posible un abordaje discursivo que permite fundamentar las interpretaciones de las problemáticas y de los conflictos sociales a través de evidencias lingüísticas (Menéndez, 2009). En este sentido y en la línea de Fairclough (1992), el análisis del discurso permite dar cuenta del rol del discurso como un proceso dinámico más que como un producto y como el dominio en el cual tienen lugar los procesos sociales. Así, considero que es una puerta de acceso a la reflexión y al pensamiento crítico, para desestabilizar los prejuicios histórica y sutilmente establecidos. En otras palabras, creo que el análisis del discurso puede colaborar a descubrir y a analizar críticamente las voces internas con las que un sujeto dialoga en los diferentes contextos en los que desarrolla sus prácticas profesionales cotidianas, ¿de dónde vienen esas voces?, ¿a quién o a quiénes representan? y, sobre todo, ¿a qué acciones nos conducirán?

Recebido em: setembro de 2009 Aprovado em: dezembro de 2009 marianacmarch@yahoo.es 


\section{Referencias Bibliográficas}

Augé, M. Los "no lugares". Espacios del anonimato. Una antropología de la sobremodernidad. Barcelona: Gedisa, 1993.

Bajtín, M. Estética de la creación verbal. México. Siglo XXI, 1982.

Bauman, Z. La globalización. Consecuencias humanas. Buenos Aires: Fondo de Cultura Económica de la Argentina S.A, 1999.

Boron, A. Estado, capitalismo y democracia en América Latina. Buenos Aires: CLACSO, 2003.

Fairclough, N. Discourse and Social Change. Cambridge. Polity Press. Blackwell Publisher, 1992.

Fairclough, N. Critical Discourse Analysis: the Critical Study of Language. London: Longman, 1995.

Fidel, C. Orientación y peculiaridades de la política económica, social y habitacional en la Argentina. La década del 90. En: Cuenya, B., Fidel, C. y Herzer, H. (coords.) Fragmentos sociales: problemas urbanos de la Argentina. Buenos Aires: Ediciones Siglo XXI, 2004, pp. 75-87.

Guba, E. G. y Lincoln Y. S. Competing Paradigms in Qualitative Research. En: Denzin, N. K. y Lincoln, Y. S (eds.) The Landscape of Qualitative Research. Theories and Issues. Thousand Oaks, CA: Sage, 1998, pp. 195-220.

Hymes, D. Models of Interaction of Language and Social Life. En: Gumperz, J. y Hymes, D. (eds.) Directions in Sociolinguistics: The Ethnography of Communication. Oxford/New York: Basil Blackwell, 1986, pp. 35-71.

Jameson, F. El posmodernismo o la lógica cultural del capitalismo avanzado. Buenos Aires: Paidós, 2005.

Kaplan, N. Nuevos desarrollos en el estudio de la evaluación en el lenguaje: la Teoría de la Valoración. En: Boletín de Lingüística, 22: 52-78, 2004.

Krmpotic, C. y Allen, I. Trayectoria familiar, ciclos políticos y bienestar. Buenos Aires: Espacio, 2003.

Lavandera, B. Decir y aludir: una propuesta metodológica. En: Filología 19/2. Buenos Aires. Instituto de Filología y Literaturas Hispánicas "Dr. Amado Alonso", 1985, pp. 21-31.

Malanca, P. y Repetto, S. Personas en situación de calle en la Ciudad de Buenos Aires: El abordaje desde un Programa Social de Emergencias. Programa Buenos 
Aires Presente. Secretaria de Desarrollo Social. Gobierno de la Ciudad de Buenos Aires, ms, 2003.

Marchese, M. C. La construcción discursiva del beneficiario en el discurso del Estado. El caso de las políticas de empleo y de vivienda. En: Actas Digitales de las V Jornadas de Sociología de la UNLP. Cambios y continuidades sociales y políticas en Argentina y la región en las últimas décadas. Departamento de Sociología, Facultad de Humanidades y Ciencias de la Educación. Universidad Nacional de La Plata, 2008a.

Marchese, M. C. La construcción discursiva del trabajo en las políticas estatales de empleo implementadas durante el periodo 1993-1999. En: Actas Digitales del III Congreso Internacional Transformaciones culturales. Debates de la teoría, la crítica y la lingüística. Facultad de Filosofía y Letras. Universidad de Buenos Aires. Buenos Aires, 2008b.

Marchese, M. C. A critical analysis of the housing policies aimed at the extremely poor. The case of the Social Development Secretariat of Buenos Aires City. En: Lorenzo- Dus, N. (ed.) Spanish at Work. Institutional Discourse in the Spanish-Speaking World. Basingstoke, England: Palgrave Macmillan, en prensa a.

Marchese, M. C. Una vivienda en nombre de la Ley. En: Discurso, pobreza y exclusión en América Latina. Santiago de Chile: Cuarto Propio, en prensa b.

Menéndez, S. M. ¿Qué es una estrategia discursiva? En: Santos, S. y Panesi, J. (comp.) Actas digitales del Congreso Internacional: Debates Actuales. Las teorías críticas de la literatura y la lingüística. Buenos Aires: Facultad de Filosofía y Letras, Universidad de Buenos Aires, 2005.

Menéndez, M. S. Historiografía lingüística y análisis del discurso: las relaciones necesarias. En: Revista argentina de historiografía lingüística (1)1: 50-66, 2009. Disponible en: http://www.rahl.com.ar/Revistas/I\%20-\%202009/menendez-RAHL-(1)2009.pdf. Fecha de consulta: mayo de 2009.

Mingo, G. (dir.) Pobreza Urbana: discursos y sujetos. Buenos Aires: Espacio Editorial, 2006.

Pardo, M. L. Derecho y Lingüística. Cómo se juzga con palabras. Buenos Aires: Nueva Visión, 1996.

Pardo, M. L. La estetización y espectacularización de la pobreza: análisis crítico del discurso posmoderno televisivo en la Argentina. En: Ortiz, T. E. y Pardo, M. L. (coords.) Desigualdades sociales y Estado. Un estudio multidisciplinar desde la posmodernidad. Buenos Aires: Departamento de Publicaciones Facultad de Derecho, Universidad de Buenos Aires y A3 Plus, 2008a, pp. 139-164. 
Pardo M. L. Una metodología para la investigación lingüística del discurso. En: Pardo, M. L. (ed.) El discurso sobre la pobreza en América Latina. Santiago de Chile: Frasis, 2008b, pp. 55-78.

Pardo M. L. La teoría de la Tonalización y la de la Valoración: dos teorías complementarias, (en este mismo volumen).

Pardo Abril, N. Cómo hacer Análisis Crítico del Discurso. Una perspectiva Latinoamericana. Santiago de Chile: Frasis, 2007.

Resende, V. M. y Ramalho, V. Análise de discurso crítica. São Paulo: Editora Contexto, 2006.

Reynals, C. y Redon, C. Programa de radicación de villas de Capital Federal, en: Cambios: municipio y políticas públicas. Buenos Aires: Subsecretaría de Programación de la Municipalidad de Buenos Aires, 1991, pp. 52-59.

Sarlo, B. Escenas de la vida Posmoderna. Buenos Aires: Ariel, 1994.

Sklair, S. Sociología del sistema global. El impacto socioeconómico y político de las corporaciones trasnacionales, traducción de María Laura Pardo. Barcelona: Gedisa, 2003.

van Dijk, T. A. Principles of critical discourse analysis. En: Discourse \& Society (4)2. London: Sage, 1993, pp. 249-283.

White P. R. R. Un recorrido por la Teoría de la Valoración. 1999, Disponible en: http://www.grammatics.com/appraisal/SpanishTranslation-AppraisalOutline. pdf. Fecha de consulta: mayo de 2009.

Wodak, R. El enfoque histórico del discurso. En: Wodak, R. y Meyer M. (comp.) Métodos de Análisis Crítico del Discurso. Barcelona: Gedisa, 2003, pp. 101-142.

Wodak, R. y Meyer, M. (comp.) Métodos de Análisis Crítico del Discurso. Barcelona: Gedisa, 2003. 